\title{
Arterial blood flow characteristics in central retinal vein occlusion and effects of panretinal photocoagulation treatment: an investigation by colour Doppler imaging
}

\begin{abstract}
Avni Murat Avunduk, Hasan Dinç, Zerrin Kapıcıoğlu, Şaban Uğurlu, Volkan Dayanır, Ercan Korkmaz
\end{abstract}

\begin{abstract}
Aims-To determine whether an increase in vascular resistance in the central retinal and ophthalmic arterial circulations contributes to the development of central retinal vein occlusion (CRVO), or haemodynamic alterations in central retinal and ophthalmic arteries occur secondary to the vein occlusion as increased intravascular pressure is transferred through the capillary bed to the arterial side and the effect of panretinal photocoagulation treatment on these circulations in ischaemic cases.
\end{abstract}

Methods-The ophthalmic and central retinal arteries of the affected and nonaffected eyes of 20 patients with nonischaemic CRVO, 13 patients with ischaemic CRVO, and 22 control subjects were investigated by colour Doppler imaging. Panretinal photocoagulation (PRP) treatment was applied to the eyes with ischaemic CRVO. Maximum and minimum blood flow velocities, and resistivity indexes were calculated in the affected and healthy eyes of patients and in the control eyes.

Results-Average blood flow velocity in the central retinal and ophthalmic arteries of patients with non-ischaemic CRVO did not differ from their fellow eyes, but a significantly lower average blood flow velocity was found in the ophthalmic and central retinal arteries of the patients with ischaemic CRVO compared with their fellow eyes. Patients with ischaemic CRVO had significantly lower blood flow velocities in their ophthalmic and central retinal arteries than non-ischaemic cases that were further reduced following PRP treatment.

Conclusion-This study suggests that impaired arterial blood flow observed in patients with CRVO may be partly related to secondary changes in the retrobulbar arterial circulation as a result of enhanced arterial resistance following CRVO. These data also demonstrate that PRP treatment decreases retinal and ophthalmic blood flow velocities in patients with ischaemic CRVO.

(Br f Ophthalmol 1999;83:50-53)

Central retinal vein occlusion (CRVO) is a common retinal vascular disorder and may lead to the development of a blind and painful eye. ${ }^{1}$ The pathogenesis of the disease has not been completely understood and there has been much debate about the involvement of the arterial and venous circulations in the disorder.$^{2-4}$ Histologically, the primary cause of CRVO is thrombosis of the central retinal vein at the level of or posterior to the lamina cribrosa sclerae. ${ }^{5}$ Two forms of the disease are recognised: "non-ischaemic," "partial," or "hyperpermeable" form, and severe, "ischaemic," "total," or "haemorrhagic" form. The latter has a worse prognosis, with poor visual recovery and more frequent complications-for example, rubeosis iridis and neovascular glaucoma. $^{6-10}$ Management of ischaemic CRVO is aimed at the prediction of complications and preventive treatment by means of destructive panretinal photocoagulation. ${ }^{11}{ }^{12}$ However, the pathogenesis of CRVO is not fully understood. Some researchers believe that retinal arterial disease plays a role in the development of CRVO, but sufficient information is not available about arterial blood flow in eyes with CRVO. ${ }^{13-15}$

This study was designed to examine the blood flow velocities of the central retinal and ophthalmic arteries of the affected and nonaffected fellow eyes in patients with either ischaemic and non-ischaemic CRVO and to determine the effect of panretinal photocoagulation treatment on these values. For this purpose, we used colour Doppler imaging to study ophthalmic and central retinal arteries. Panretinal photocoagulation was applied to ischaemic patients and blood flow analyses repeated after treatment. The results were compared with healthy fellow eyes and normal subjects. One of the main aims of the study was to determine whether an increase in vascular resistance in the retrobulbar arterial circulation contributes to the development of CRVO or haemodynamic alterations in central retinal ophthalmic artery occur secondary to the vein occlusion which transfers increased intravascular pressure through the capillary bed to the arterial side. Colour Doppler imaging is a noninvasive, reproducible, and easily applied technique that is shown to be useful in evaluating haemodynamic changes in several orbital and retinal vascular diseases. ${ }^{16-19}$

Materials and methods

Thirty three patients with CRVO of 3 months' duration or less were enrolled into the study group. Fifteen of the patients with CRVO were female and 18 were male. The average age of the patients was 64.6 (SD 2.1) years. All 
Table 1 Results of velocimetry measurements and resistivity indexes * (mean (SD))

\begin{tabular}{|c|c|c|c|c|c|c|}
\hline & \multicolumn{3}{|c|}{ Ophthalmic artery } & \multicolumn{3}{|c|}{ Central retinal artery } \\
\hline & PSVt & $E D V t$ & $R I \ddagger$ & PSVt & $E D V t$ & $R I \ddagger$ \\
\hline \multicolumn{7}{|l|}{ Ischaemic $(n=13)$} \\
\hline \multicolumn{7}{|l|}{ Eye with } \\
\hline CRVO & $28.7(9.8)$ & $5.9(2.5)$ & $79.4(11.2)$ & $6.9(1.8)$ & $1.9(0.9)$ & $73.2(10.8)$ \\
\hline Fellow eye & $34.7(9.6)$ & $7.9(2.8)$ & $64.8(10.4)$ & $8.1(2.3)$ & $2.6(1.2)$ & $60.5(11.0)$ \\
\hline Following PRP & $24.3(8.7)$ & $5.0(2.6)$ & $82.4(13.6)$ & $6.3(1.7)$ & $1.4(0.8)$ & $76.9(13.4)$ \\
\hline \multicolumn{7}{|c|}{ Non-ischaemic $(\mathrm{n}=20)$} \\
\hline \multicolumn{7}{|l|}{ Eye with } \\
\hline CRVO & $34.6(8.6)$ & $8.0(2.5)$ & $65.2(10.9)$ & $8.2(2.2)$ & $2.5(1.3)$ & $60.6(11.3)$ \\
\hline Fellow eye & $34.5(9.1)$ & $7.9(2.7)$ & $64.9(11.1)$ & $8.3(2.3)$ & $2.6(1.2)$ & $60.8(11.2)$ \\
\hline Controls $(n=22)$ & $38.6(10.1)$ & $9.2(3.5)$ & $58.4(9.5)$ & $9.4(3.1)$ & $4.1(1.9)$ & $54.7(9.7)$ \\
\hline
\end{tabular}

${ }^{\star}$ All values are within the $95 \%$ of confidence limits; $† \mathrm{~cm} / \mathrm{s}$; $\neq$ percentage; PSV = peak systolic velocity; $\mathrm{EDV}=$ end diastolic velocity; $\mathrm{RI}=$ resistivity index $; \mathrm{CRVO}=$ central retinal vein oclusion; $\mathrm{PRP}=$ panretinal photocoagulation

patients had a unilateral involvement. Twenty of them had systemic hypertension (14 in the non-ischaemic group and six in the ischaemic group) and two had diabetes mellitus (both of them in the non-ischaemic group). Three (two in the non-ischaemic group and one in the ischaemic group) of the patients had open angle type glaucoma that was controlled by medical therapy at the time of colour Doppler imaging. All the patients included in this study had characteristic fundus findings of CRVO. All had dilated and tortuous retinal veins, retinal haemorrhages, optic disc oedema, and macular oedema. Of the 33 patients 20 had non-ischaemic and 13 had ischaemic type CRVO. The average ages of non-ischaemic and ischaemic patients were 64.8 (1.9) and 64.4 (2.0) respectively. Patients were defined as having a non-ischaemic CRVO if they had fewer than 10 cotton wool spots in a $45^{\circ}$ photograph of the posterior pole, less than 10 optic disc areas of retinal capillary non-perfusion on fluorescein angiography, and no neovascularisation of the iris. Patients were defined as having an ischaemic CRVO if they had greater than 10 cotton wool spots in a $45^{\circ}$ photograph of the posterior pole, 10 or more optic disc areas of retinal capillary non-perfusion on fluorescein angiography, and/or neovascularisation of the iris. Panretinal argon laser photocoagulation at green wavelength was applied to all patients who had an ischaemic type CRVO, using a spot size of $500 \mu \mathrm{m}$ with $250-700 \mathrm{~mW}$ energy, and 0.1 second duration in varying numbers of $1500-1800$ spots as needed.

Twenty two healthy volunteers who had undergone colour Doppler imaging were chosen for the control group. Healthy volunteers

Table 2 Results of statistical analyses for velocimetry measurements and resistivity indexes

\begin{tabular}{|c|c|c|c|c|c|c|}
\hline & \multicolumn{3}{|c|}{ Ophthalmic artery } & \multicolumn{3}{|c|}{ Central retinal artery } \\
\hline & PSV & $E D V$ & $R I$ & PSV & $E D V$ & $R I$ \\
\hline Is $\mathrm{CRVO}$ - $\mathrm{fel} \dagger$ & $\mathrm{p}<0.05$ & $\mathrm{p}<0.05$ & $\mathrm{p}<0.05$ & $\mathrm{p}<0.05$ & $\mathrm{p}<0.05$ & $\mathrm{p}<0.05$ \\
\hline Is $\mathrm{CF}$ & $\mathrm{p}<0.05$ & $\mathrm{p}<0.05$ & $\mathrm{p}<0.05$ & $\mathrm{p}<0.05$ & $\mathrm{p}<0.05$ & $\mathrm{p}<0.05$ \\
\hline Is $\mathrm{CRVO}$-non-is CRVO $\ddagger$ & $\mathrm{p}<0.05$ & $\mathrm{p}<0.05$ & $\mathrm{p}<0.05$ & $\mathrm{p}<0.05$ & $\mathrm{p}<0.05$ & $\mathrm{p}<0.05$ \\
\hline Non-is CRVO-felt & $\mathrm{p}>0.05$ & $p>0.05$ & $p>0.05$ & $\mathrm{p}>0.05$ & $p>0.05$ & $\mathrm{p}>0.05$ \\
\hline Non-is CRVO-controls $\ddagger$ & $\mathrm{p}<0.05$ & $\mathrm{p}<0.05$ & $\mathrm{p}<0.05$ & $\mathrm{p}<0.05$ & $\mathrm{p}<0.05$ & $\mathrm{p}<0.05$ \\
\hline Is CRVO-controls $\ddagger$ & $\mathrm{p}<0.05$ & $\mathrm{p}<0.05$ & $\mathrm{p}<0.05$ & $\mathrm{p}<0.05$ & $\mathrm{p}<0.05$ & $\mathrm{p}<0.05$ \\
\hline Fel Is CRVO-controls $\ddagger$ & $\mathrm{p}<0.05$ & $\mathrm{p}<0.05$ & $\mathrm{p}<0.05$ & $\mathrm{p}<0.05$ & $\mathrm{p}<0.05$ & $\mathrm{p}<0.05$ \\
\hline Fel Non-is CRVO_controls $\ddagger$ & $\mathrm{p}<0.05$ & $\mathrm{p}<0.05$ & $\mathrm{p}<0.05$ & $\mathrm{p}<0.05$ & $\mathrm{p}<0.05$ & $\mathrm{p}<0.05$ \\
\hline Fel Is CRVO-fel non-is CRVO $\ddagger$ & $\mathrm{p}>0.05$ & $\mathrm{p}>0.05$ & $\mathrm{p}>0.05$ & $\mathrm{p}>0.05$ & $\mathrm{p}>0.05$ & $p>0.05$ \\
\hline
\end{tabular}

$\mathrm{CRVO}=$ central retinal vein occlusion; $\mathrm{PRP}=$ panretinal photocoagulation; $\mathrm{Fel}=$ fellow eyes; Is $=$ ischaemic , non-is $=$ non-ischaemic $; \dagger=$ statistical analyses were performed by using Wilcoxon matched pair signed rank test; $\ddagger=$ statistical analyses were performed by using Mann-Whitney U test. had no clinical evidence of retinal or systemic vascular disease. Twelve of the volunteers were male. The average age of the volunteers was 64.1 (1.8) years.

A Hitachi 515 Euba ultrasonic colour pulse Doppler unit with a $7.5 \mathrm{MHz}$ linear phase transducer (Tokyo, Japan) was used to examine the retrobulbar circulation in all subjects as described by Lieb et al. ${ }^{20}$ Informed consent was obtained from each patient and volunteer. Peak systolic (PSV) $(\mathrm{cm} / \mathrm{s})$, and end diastolic blood flow velocities (EDV) were measured from the time velocity waveforms of the central retinal (CRA), and ophthalmic arteries (OA) during the spectral analysis by averaging the readings from three waveforms. Maximum working range (within the $95 \%$ of confidence limit) of our machine for a $7.5 \mathrm{Mz}$ probe is given between the range of $1 \mathrm{~cm} / \mathrm{s}$ and $118 \mathrm{~cm} / \mathrm{s}^{21}$ Resistivity indexes (RI) were calculated according to the formula:

$$
\text { Resistivity index }=\frac{\text { PSV }- \text { EDV }}{\text { PSV }}
$$

All measurements were performed by the same operator. Low pulse repeat frequency was used to detect slow blood flow. Flow towards, and away from the transducer was depicted as red and blue respectively. During the eye examination, the operator attempted to align the ultrasound beam parallel to the vessel under observation and its blood flow.

Multiple comparisons were performed for each blood vessel. Firstly, the involved eye of each patient with CRVO was compared with the patient's fellow healthy eye using Wilcoxon matched pair signed rank test. In addition, the involved and healthy eyes of patients with CRVO were compared with the eyes of healthy volunteers using the Mann-Whitney U test. Involved and healthy eyes of patients with ischaemic CRVO were compared with involved and healthy eyes of non-ischaemic patients using Mann-Whitney U test. Finally, values obtained in the pretreatment stage of the involved eyes of ischaemic patients were compared with values obtained 1 month after panretinal photocoagulation treatment using paired Wilcoxon matched pair signed rank test. Statistical significance was accepted as $\mathrm{p}<0.05$.

\section{Results}

The blood flow velocities, and resistivity indexes obtained from affected and unaffected eyes of patients with CRVO, and healthy volunteers for the central retinal and ophthalmic arteries are shown in Tables 1 and 2. All the obtained values were within $95 \%$ of the confidence limit of the colour Doppler unit.

Comparison of the eyes with ischaemic CRVO with healthy fellow eyes, eyes with nonischaemic CRVO, and controls showed significantly lower velocities in the central retinal and ophthalmic arteries in eyes with ischaemic CRVO. A significantly higher average resistivity indexes was found in the central retinal and ophthalmic arteries of patients with ischaemic CRVO compared with their fellow eyes, eyes with non-ischaemic CRVO, and healthy volunteers. In the non-ischaemic cases, arterial 
blood flow velocities and resistivity indexes did not differ significantly from their healthy fellow eyes. However, significantly lower PSV, EDV, and higher resistivity indexes were found in the central retinal, and ophthalmic arterial circulations of the patients with non-ischaemic CRVO compared with healthy controls.

Comparison of the unaffected eyes of patients with ischaemic and non-ischaemic CRVO with each other did not give any significant difference either for flow velocities or resistive indexes.

Significantly lower PSV and EDV, and higher resistive indexes were present in the central retinal and ophthalmic arteries of the healthy fellow eyes of the patients with CRVO than the controls $(p<0.05$ for all values).

Arterial blood flow velocities in the eyes of patients with ischaemic CRVO were found to be significantly lower, and resistivity indexes higher following PRP treatment compared with their pretreatment values.

\section{Discussion}

Our data demonstrated a reduction of blood flow velocities in the central retinal and ophthalmic arteries in patients with CRVO which was more marked in the ischaemic cases compared with non-ischaemic patients. Haemodynamic abnormalities in the retrobulbar arterial circulation of patients with CRVO was reported previously. ${ }^{22}$ In patients with CRVO, substantial arterial blood flow disturbances, such as the reduction of the retinal artery peak systolic flow and absence of diastolic flow were also reported by different researchers. ${ }^{23}$ However, in both studies the authors did not compare the measurement obtained from ischaemic patients with nonischaemic cases. Baxter and Williamson performed further studies on the blood flow velocities in ophthalmic and retinal arteries of patients with CRVO and found that reduction of the blood flow velocities in these arteries did not correlate with the ischemia. ${ }^{24}{ }^{25}$ Based on their data, they suggested that a generalised increase in vascular resistance in the retrobulbar arterial circulation may predate and contribute to the development of CRVO. ${ }^{24}{ }^{25}$ However, it is also possible that haemodynamic alterations in central retinal artery and ophthalmic artery may occur secondary to the vein occlusion as a result of increased intravascular pressure transferred through the capillary bed to the arterial side. ${ }^{3}$ Our findings suggest this latter mechanism may also play a role in the retrobulbar circulation abnormalities observed in CRVO. We found lower PSV and EDV, and higher RI values in the central retinal and ophthalmic arteries of patients with ischaemic CRVO compared with non-ischaemic CRVO patients. Furthermore, patients with nonischaemic type CRVO had lower PSV and EDV, and higher RI values in their central retinal and ophthalmic arteries of both affected and healthy fellow eyes compared with controls. Blood flow characteristics in the central retinal and ophthalmic arteries of the affected eyes of non-ischaemic patients did not differ significantly from their healthy fellow eyes.
Similarly, Keyser et al reported a higher average RI, and lower average PSV in the central retinal, and ophthalmic arteries of the affected and unaffected healthy eyes of patients with CRVO compared with healthy volunteers, but they did not group their patients according to ischaemia. ${ }^{22}$

Patients with ischaemic CRVO had significantly lower PSV and EDV, and higher RI values in the central retinal and ophthalmic arteries of the affected eyes than their healthy fellow eyes, non-ischaemic eyes, and controls. Our data on ophthalmic artery measurements do not correlate with the previous works of Baxter and Williamson, since they did not report a velocity difference between the ophthalmic arteries of the affected eyes of ischaemic CRVO patients compared with the affected eyes of non-ischaemic cases. ${ }^{25}$ There is no reasonable explanation for this difference since both studies had similar methods and patient selection criteria. We propose that abnormalities observed in the retrobulbar arterial circulation of the ischaemic CRVO patients compared with non-ischaemic cases may be related to secondary changes in arterial blood flow following CRVO owing to increased vascular resistance. On the other hand, several haemodynamic abnormalities were present in the arterial circulation of the healthy and affected eyes of non-ischaemic patients with CRVO suggesting that some haemodynamic abnormalities in the retrobulbar arterial circulation may cause and predate the clinical onset of CRVO. It has been suggested that the resulting arterial insufficiency as a result of arteriosclerosis or atherosclerosis may contribute circulatory stasis in central retinal vein. ${ }^{2}$ In addition, the thickening and increased rigidity of the central retinal artery wall as a result of atherosclerosis and arteriosclerosis subsequently may impinge upon the lumen of the central retinal vein in the area of the lamina cribrosa, contributing to venous thrombus formation. ${ }^{5}$ The available evidence suggests that the site of venous occlusion in CRVO is probably within or just posterior to the lamina cribrosa. ${ }^{7}$ Thus, it seems likely that both of the above mentioned mechanisms play a role in causing the haemodynamic abnormalities seen in the central retinal artery of the eyes with CRVO. Haemodynamic abnormalities observed in the retrobulbar arterial circulation, central retinal artery, and ophthalmic artery of the healthy fellow eyes of patients with CRVO may predispose these clinically healthy eyes to CRVO and may explain the risk of bilateral disease in $6 \%-16 \% .^{76-28}$

We found that PRP treatment further impaired retinal and ophthalmic circulation in patients with ischaemic CRVO. Following PRP treatment, lower PSV, EDV, and higher resistive indexes were found in the central retinal and ophthalmic arteries of the patients with ischaemic CRVO than their pretreatment values. In a recent study, peak systolic blood flow velocities were found to be significantly lower in central retinal veins of PRP treated diabetic patients than preretinopathy group. ${ }^{29}$ A lower mean PSV in the central retinal 
arteries of the PRP treated patients compared with patients with preretinopathy was reported by the same authors, but the difference did not reach a significant level. However, the authors did not compare the blood velocities of their patients in the pretreatment stage with posttreatment values. ${ }^{29}$ In another study concerning patients with proliferative diabetic retinopathy, following PRP treatment a significant decrease in retinal volumetric blood flow rate and an increase in the retinal vascular regulatory response to hyperoxia were found by using bidirectional laser Doppler velocimetry and monochromatic fundus photography techniques. ${ }^{30}$ The results of our study suggests that panretinal photocoagulation treatment has the same effect on central retinal and ophthalmic arterial circulation of ischaemic type CRVO as in diabetic retinopathy.

In conclusion, impaired arterial blood flow observed in patients with CRVO may partly be related to secondary changes in the retrobulbar arterial circulation due to enhanced arterial resistance following CRVO. Panretinal photocoagulation treatment further impaired blood flow in central retinal and ophthalmic arteries of patients with ischaemic CRVO. Colour Doppler imaging may be a useful technique for assessing central retinal and ophthalmic arterial circulation abnormalities observed in CRVO and further studies using this technique may be helpful for better understanding of the pathogenesis of the disease.

1 Hayreh SS. Occlusion of central retinal vessels. $\mathrm{Br} \mathcal{F}$ Ophthalmol 1965;49:426-45.

2 Hayreh SS, van Heuven WAJ, Hayreh MS. Experimental Hayreh SS, van Heuven WAJ, Hayreh MS. Experimental retinal vascular occlusion. I. Pathogenesis of centr
vein occlusion. Arch Ophthalmol 1978;96:311-23.

3 Fujino T, Curtin VT, Norton EWD. Experimental central retinal vein occlusion. A comparison of intraocular and extraocular occlusion. Arch Ophthalmol 1969;81:395-406.

4 Jorizzo PA, Klein ML, Shults WT, et al. Visual recovery in combined central retinal artery and central retinal vein occlusion. Am F Ophthalmol 1987;104:358-63.

5 Green WR, Chan CC, Hutchins GM, et al. Central retinal vein occlusion: a prospective histopathological study of 29 eyes in 28 cases. Trans Am Ophthalmol Soc 1981;79:371422.

6 Sinclair SH, Gagourdas ES. Prognosis for rubeosis iridis following central retinal vein occlusion. $\mathrm{Br} \mathcal{F}$ Ophthalmol 1983;67:137-42.

7 Qiunlain PM, Elman MJ, Bhatt AK, et al. The natural course of central retinal vein occlusion. Am $\mathcal{f}$ Ophthalmol 1990;110:118-23.
8 Hayreh SS, Rojas P, Podhajsky P, et al. Ocular neovascularization with retinal vascular occlusion. III. Incidence of ocular neovascularization with
thalmology 1983;90:488-506.

9 Zegarra H, Gutman FA, Conforto J. The natural course of central retinal vein occlusion. Ophthalmology 1979;86: 1931-42.

10 Magargal LE, Brown GC, Augsburger JJ, et al. Neovascular glaucoma following central retinal vein obstruction. Ophthalmology 1981;88:1095-101.

11 May DR, Klein ML, Peyman GA. A prospective study of xenon arc photocoagulation for central retinal vein occlusion. Br f Ophthalmol 1976;60:816-8.

12 Laatikainen L, Kohner EM, Khoury D, et al. Panretinal photocoagulation in central retinal vein occlusion: a randomised controlled clinical study. $\mathrm{Br} \mathcal{F}$ Ophthalmol 1977;61:741-53.

13 Hayreh SS. Pathogenesis of occlusion of the central retinal vessels. Am f Ophthalmol 1971;72:998-1011.

14 Paton A, Rubinstein K, Smith VH. Arterial insufficiency in retinal venous

15 Hayreh SS. So called 'central retinal vein occlusion.' I. Pathogenesis, terminology, clinical features. OphthalmoPathogenesis, termin

16 Lieb WE. Colour Doppler ultrasonography of eye and orbit. Curr Opin Ophthalmol 1993;4:68-75.

17 Aburn NS, Sergott RC. Orbital colour Doppler imaging. Eye 1993;7:639-47.

18 Regillo CD, Sergott RC, Ho AC, et al. Hemodynamic alteration in the acute retinal necrosis syndrome. Ophthalmology 1993;100:1171-6.

19 Ho AC, Lieb WE, Flaharty PM, et al. Colour Doppler imaging of the ocular ischemic syndrome. Ophthalmology 1992;99:1453-62.

20 Lieb WE, Cohen SM, Merton DA, et al. Colour Doppler maging of the eye and orbit: technique and normal vascular anatomy. Arch Ophthalmol 1991;109:527-31.

21 Hitachi Medical Corporation. Instruction manual for ultrasound scanner with digital scan converter model Euba-515. p 42. Tokyo: Hitachi, 1994

22 Keyser BJ, Flaharty PM, Sergott RC, et al. Color Doppler imaging of arterial blood flow in central retinal vein occlusion. Ophthalmology 1994;101:1357-61.

23 Pichot O, Gonzalvez B, Franco A, et al. Colour Doppler ultrasonography in the study of orbital and ocular vascular diseases. F Fr Ophtalmol 1996;19:19-31.

24 Williamson TH, Baxter GM. Central retinal vein occlusion, an investigation by colour Doppler imaging. Blood velocity characteristics and prediction of iris neovascularization. Ophthalmology 1994;101:1362-72.

25 Baxter GM, Williamson TH. The value of serial Doppler maging in central retinal vein occlusion:correlation with visual recovery. Clin Radiol 1996;51:11-4.

26 Hayreh SS. Classification of central retinal vein occlusions. Ophthalmology 1983;90:458-74.

27 Rubistein K, Jones EB. Retinal vein occlusion: long-term prospects: 10 years' follow-up of 143 patients. $\mathrm{Br} f$ Ophthalmol 1976;60:148-50.

28 Elman MJ, Bhatt AK, Quinlain PM, et al. The risk for systemic vascular disease and mortality in patients with central retinal vein occlusion. Ophthalmology 1990;97: $1543-8$

29 Güven D, Özdemir H, Hasanreisodlu B. Hemodynamic alterations in diabetic retinopathy. Ophthalmology 1996; 103:1245-9.

30 Grunwald JE, Brucker AJ, Petrig BL, et al. Retinal blood flow regulation and the clinical response panretinal photocoagulation in proliferative diabetic retinopathy. Ophthalmology 1989;96:1518-22. 